\title{
Intoxicação natural por Ipomoea asarifolia (Convolvulaceae) em búfalos na Ilha de Marajó, Pará ${ }^{1}$
}

\author{
José Diomedes Barbosa ${ }^{2 *}$, Carlos Hubinger Tokarnia ${ }^{3}$, Tatiane Teles Albernaz ${ }^{2}$, \\ Carlos Magno Chaves Oliveira ${ }^{2}$, Natália da Silva e Silva² ${ }^{2}$, José Alcides Sarmento \\ da Silveira ${ }^{2}$, Alessandra dos S. Belo Reis ${ }^{2}$ e Danillo Henrique da Silva Lima ${ }^{2}$
}

\begin{abstract}
Barbosa J.D., Tokarnia C.H., Albernaz T.T., Oliveira C.M.C., Silva N.S., Silveira J.A.S., Belo Reis A.S. \& Lima D.H.S. 2012. [Natural poisoning by Ipomoea asarifolia (Convolvulaceae) in buffaloes on the Marajó Island, Pará, Brazil.] Intoxicação natural por Ipomoea asarifolia (Convolvulaceae) em búfalos na Ilha de Marajó, Pará. Pesquisa Veterinária Brasileira 32(9):869-871. Faculdade de Medicina Veterinária, Universidade Federal do Pará, Campus Castanhal, Rua Maximino Porpino 1000, Castanhal, PA 68743-080, Brazil. E-mail: diomedes.barbosa.neto@gmail.com

Epidemiological aspects and clinical signs of natural poisoning by Ipomoea asarifolia in buffaloes are described. The poisoning was diagnosed in four buffaloes on three different farms, in the County of Cachoeira do Arari, Marajó Island, Pará, and occurred in November-December, a period of drought and severe pasture shortage. The clinical signs were of the central nervous system, as unsteady gait, hypermetria, severe muscular tremors, falling down in unusual positions, nystagmus and marked excitement, signs that turned more severe after movement. Based on the epidemic aspects, clinical signs and absence of histopatological changes, poisoning by Ipomoea asarifolia was diagnosed.
\end{abstract}

INDEX TERMS: Poisonous plants, Ipomoea asarifolia, Convolvulaceae, plant poisoning, buffaloes.

RESUMO.- Descrevem-se os aspectos epidemiológicos e os sinais clínicos dos primeiros casos de intoxicação natural por Ipomoea asarifolia em búfalos. A doença foi diagnosticada em quatro bubalinos de três diferentes propriedades, no município de Cachoeira do Arari, Ilha de Marajó, PA, e ocorreu nos meses de novembro e dezembro, o período mais seco do ano nesta região e de escassez de alimento. Os sinais clínicos observados foram relacionados ao sistema nervoso central, como andar trôpego, hipermetria, acentuados tremores musculares, queda ao solo em posições incomuns, nistagmo e marcada excitação, sinais que se agravavam após movimentação. Baseado nos aspectos epidemiológicos, sinais clínicos e na ausência de leões histopatológicas, concluiu se tratar de intoxicação por Ipomoea asarifolia.

\footnotetext{
${ }^{1}$ Recebido em 2 de abril de 2012.

Aceito para publicação em 17 de maio de 2012

${ }^{2}$ Faculdade de Medicina Veterinária, Universidade Federal do Pará (UFPA), Campus Universitário de Castanhal, Rua Maximino Porpino 1000, Pirapora, Castanhal, PA 68740-080, Brasil. *Autor para correspondência: diomedes.barbosa.neto@gmail.com

${ }^{3}$ Departamento de Nutrição e Pastagem, Instituto de Zootecnia, Universidade Federal Rural do Rio de Janeiro (UFRRJ), Seropédica, RJ 23890-000, Brasil.
}

TERMOS DE INDEXAÇÃO: Plantas tóxicas, Ipomoea asarifolia, Convolvulaceae, intoxicação por plantas, búfalos.

\section{INTRODUÇÃO}

Conhecida popularmente por "salsa" ou "batatarana", Ipomoea asarifolia R. et Schult. é uma planta herbácea prostrada ou trepadeira, da família Convolvulaceae, que ocorre nas margens de rios e lagos e nas praias marítimas, em terrenos abandonados, nas margens de estradas e em lugares próximos de habitações. Os animais ingerem a planta quando estão com fome, e para mostrar sinais de intoxicação tem que ingeri-la em grandes quantidades (Tokarnia et al. 2007).

Em condições naturais, foram diagnosticados casos de intoxicação em bovinos, ovinos e caprinos (Riet-Correa et al. 2003, 2006, Tortelli et al. 2008, Tokarnia et al. 2012). Experimentos com I. asarifolia foram realizados em bovinos, ovinos e caprinos (Döbereiner et al. 1960, Medeiros et al. 2003, 2004, Araújo et al. 2004, 2008, Barbosa et al. $2004,2005)$. Bubalinos mostraram-se experimentalmente também suscetíveis à intoxicação por I. asarifolia (Barbosa et al. 2005).

Embora Ipomoea asarifolia tenha sido considerada uma planta de menor importância para a região norte (Tokarnia 
et al. 2007), na Ilha do Marajó, onde há grande quantidade dessa planta, já existem relatos de intoxicação natural em bovinos (Barbosa et al. 2005, Tortelli et al. 2008) e ovinos (Tortelli et al. 2008). No entanto, apesar da grande população de bubalinos que habita a Ilha e de ser conhecida experimentalmente a toxidez da I. asarifolia para essa espécie (Barbosa et al. 2005), não existem relatos da intoxicação natural por essa planta em búfalos. 0 objetivo do presente trabalho foi relatar a ocorrência da intoxicação natural por I. asarifolia em búfalos.

\section{MATERIAL E MÉTODOS}

Os dados sobre a intoxicação por Ipomoea asarifolia nos búfalos foram obtidos através de visitas a três propriedades rurais da Ilha de Marajó, localizadas no município de Cachoeira do Arari, Pará.

Durante as visitas foi realizado um levantamento das plantas tóxicas existentes, foram examinados animais intoxicados, realizadas necropsias de dois animais gravemente doentes e eutanasiados, com coleta de material para estudos histopatológicos. Para esta última finalidade o material foi fixado em formalina a $10 \% \mathrm{e}$ processado pelos métodos rotineiros, incluído em parafina, cortado na espessura de 5 micra e corado pela hematoxilina-eosina (HE).

\section{RESULTADOS}

As visitas às propriedades na Ilha de Marajó foram realizadas durante o período seco. Na inspeção das pastagens, verificou-se escassez de forragem e presença de grandes quantidades de Ipomoea asarifolia (Fig.1) e de Ipomoea carnea subsp. fistulosa, que estavam verdes, com flores e frutos. Havia sinais de essas plantas terem sido consumidas. Nas três propriedades visitadas foram observadas quatro búfalas, com idade de dois a cinco anos, com sintomatologia nervosa. Esses sinais clínicos, principalmente visíveis após alguns minutos de movimentação dos animais, consistiam em andar trôpego, acentuados tremores musculares, hipermetria (Fig.2), queda ao solo em posições incomuns (Fig.3 e 4), nistagmo e marcada excitação. Deixados em sossego, após pouco tempo, os animais se levantavam, sem demonstrar quaisquer sintomas além de leve incoordenação. Quando não movimentados, observando-se com cuidado os búfalos afetados, às vezes podiam se constatar tremores musculares e incoordenação leves. Os achados de necropsia e os exames histopatológicos foram negativos.

Os proprietários informaram que esta doença ocorria quase todos os anos, na época de escassez de pasto e que afetava bovinos, ovinos e caprinos, porém nunca haviam visto bubalinos acometidos.

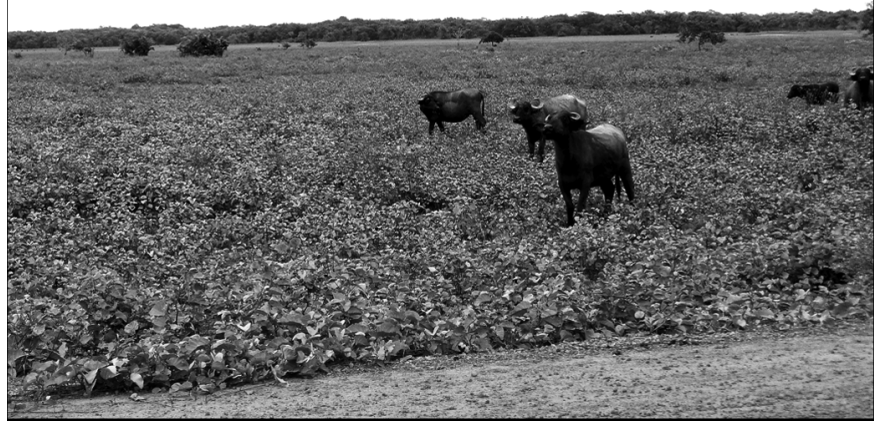

Fig.1. Búfalos em pastagem invadida por Ipomoea asarifolia. Cachoeira do Arari, Ilha de Marajó, PA.

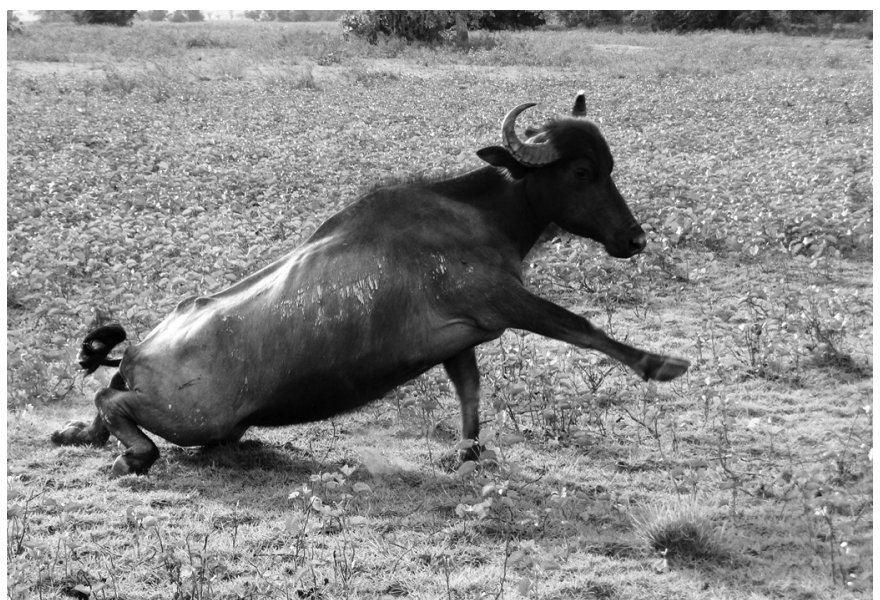

Fig.2. Intoxicação natural por Ipomoea asarifolia. Búfalo com hipermetria. Cachoeira do Arari, Ilha de Marajó, PA.
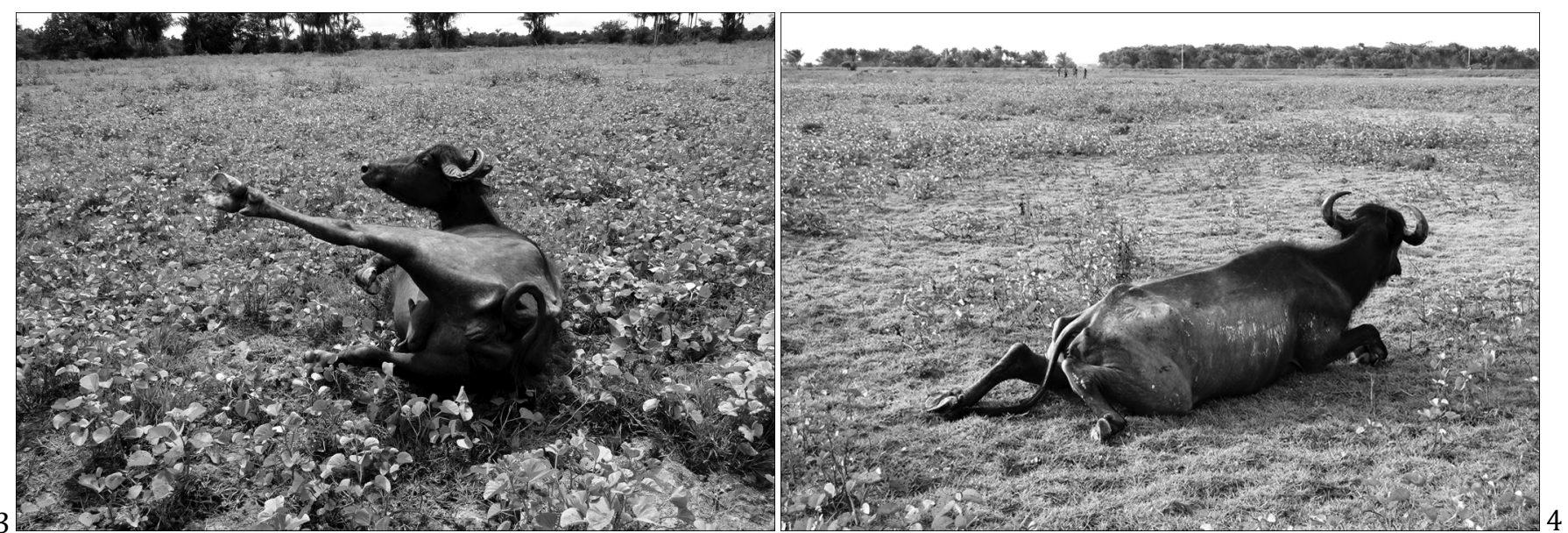

Fig.3 e 4. Queda de búfalo ao solo em posições incomuns na intoxicação natural por Ipomoea asarifolia. Cachoeira do Arari, Ilha de Marajó, PA. 


\section{DISCUSSÃO}

A substituição das pastagens nativas por Ipomoea asarifolia vem ocorrendo gradativamente, a cada ano, em muitos campos nativos na Ilha de Marajó. A presença de grande quantidade da planta na área onde permaneciam os búfalos afetados pela doença, associada à escassez de alimento, foi possivelmente o fator determinante para que os bubalinos passassem a ingerir e se intoxicar com a planta. Vale ressaltar, que no ano em que foram verificados os casos naturais da enfermidade, o período seco foi mais longo que o usual, o que agravou ainda mais a falta de alimento na Ilha de Marajó.

Os sinais clínicos apresentados pelos búfalos foram semelhantes aos descritos por Barbosa et al. (2005) na intoxicação experimental por I. asarifolia nesta espécie. No presente estudo, os sinais de incoordenação foram mais acentuados do que na intoxicação experimental.

A ausência de lesões macroscópicas e microscópicas verificada no presente estudo também foi relatada na intoxicação experimental por I. asarifolia em búfalos (Barbosa et al. 2005). Medeiros et al. (2003, 2004), Araujo et al. (2008) e Tortelli et al. (2008) também não observaram alterações histológicas nos casos de intoxicação por I. asarifolia em bovinos, caprinos e ovinos. Já Araujo et al. (2004) observaram em um cordeiro, na intoxicação natural, vacúolos na camada granular do cerebelo. Guedes et al. (2007) relataram que em dois dos cinco surtos de intoxicação por I. asarifolia em ovinos, em dois os animais não apresentaram lesões histológicas dos sistema nervoso central, porém no ovino de um surto havia vacúolos na camada granular do cerebelo com raros esferoides axonais e moderado número de células de Purkinje contendo vacúolos intracitoplasmáticos. No ovino de outro surto havia uma quantidade discreta de esferoides axonais na camada granular do cerebelo. E no ovino de ainda outro dos cinco surtos havia vacuolização das células de Purkinje e também numerosos esferoides na camada granular do cerebelo.

Riet-Correa et al. (2006) a respeito de alterações histológicas na intoxicação por I. asarifolia, informam que na histologia somente se observam lesões degenerativas das células de Purkinje em casos de longa duração.

Araujo et al. $(2003,2004)$ descrevem alterações ultraestruturais. Medeiros et al. $(2003,2004)$ e Tortelli et al. (2008) não verificaram alterações ultraestruturais em casos de intoxicação por I. asarifolia. Guedes et al. (2007) e Tortelli et al (2008) não realizaram estudos a esse respeito.

Em relação ao princípio tóxico, Medeiros et al. 2003 relatam que I. asarifolia usado em experimentos na Paraíba, continha quantidades mínimas de swansonina (menos de $0,001 \%$ ) e que as análises foram negativas para calysteginas; concluíram que I. asarifolia causa uma síndrome tremorgência devido a uma fitotoxina ou micotoxina desconhecida, e que a intoxicação por I. asarifolia não é uma doença de armazenamento. Em outra publicação, Medeiros et al. (2004) informam que I. asarifolia na Paraíba continha uma quantidade quase indetectável de swasonsonina, porém diversos calysteginas, inclusive callisteginas B2 e C1.
Opinam que novos experimentos são necessários para determinar se I. asarifolia causa doença de armazenamento. Mas Riet-Correa et al. (2006) concluíram que I. asarifolia pertence a um grupo de plantas denominadas tremorgênicas e que o princípio ativo é desconhecido.

\section{CONCLUSÕES}

Os dados epidemiológicos, associados à sintomatologia nervosa e ausência de lesões histológicas de significação, permitiram o diagnóstico de intoxicação natural por Ipomoea asarifolia em búfalos na Ilha de Marajó.

Embora os proprietários nunca tenham visto búfalos intoxicados por I. asarifolia na Ilha de Marajó e não haja relatos anteriores da intoxicação natural por essa planta em búfalos, é provável que casos de intoxicação nessa espécie estejam passando despercebidos.

\section{REFERÊNCIAS}

Araújo J.A.S., Riet-Correa F., Barros S.S., Vasconcelos J.S., Oliveira D.M., Barbosa R.C. \& Medeiros R.M.T. 2004. Intoxicação por Ipomoea asarifolia em ovinos. Pesq. Vet. Bras. 24(Supl.):6-7.

Araújo J.A.S., Riet-Correa F., Medeiros R.M.T., Soares M.P., Oliveira D.M. \& Carvalho F.K.L. 2008. Intoxicação experimental por Ipomoea asarifolia (Convolvulaceae) em caprinos e ovinos. Pesq. Vet. Bras. 28(10):488-494.

Barbosa R.C., Medeiros R.M., Riet-Correa F., Araújo J.A.S., Lima E.F. \& Vasconcelos J.S. 2004. Intoxicação experimental por Ipomoea asarifolia (salsa) em caprinos. Pesq. Vet. Bras. 24(Supl.):9-10.

Barbosa J.D., Oliveira C.M.C., Duarte M.D., Peixoto P.V. \& Tokarnia C.H. 2005. Intoxicação experimental e natural por Ipomoea asarifolia (Convolvulaceae) em búfalos e outros ruminantes. Pesq. Vet. Bras. 25(4):231-234.

Döbereiner J., Tokarnia C.H. \& Canella C.F.C. 1960. Intoxicação experimental pela "salsa" (Ipomoea asarifolia R. et Schult.). Arqs Inst. Biol. Anim., Rio de J., 3:39-57.

Guedes K.M.R., Riet-Correa F., Dantas A.F.M., Simões S.V.D., Miranda Neto E.G., Nobre V.M.T. \& Medeiros R.M.T. 2007. Doenças do sistema nervoso central em caprinos e ovinos no semi-árido. Pesq. Vet. Bras. 27(1):2938.

Medeiros R.M.T., Barbosa R.C., Riet-Correa F., Lima E.F., Tabosa I.M., Barros S.S., Gardner D.R. \& Molyneux R.J. 2003. Tremorgenic syndrome in goats caused by Ipomoea asarifolia in northeastern Brasil. Toxicon 41:993935.

Medeiros R.M.T., Riet-Correa F., Barbosa R.C., Lima E.F., Neto S.A.G. \& Tabosa I.M. 2004. Toxic plants for domestic animals in the State of Paraiba, northeastern Brazil, p.151-156. In: Acamovic T., Stewart C.S. \& Pennycott T.W. (Eds), Poisonous Plants and Related Toxins. CABI, Wallingford, UK.

Riet-Correa F., Tabosa I.M., Azevedo E.O., Medeiros R.M.T., Simões S.V.D., Dantas A.F.M., Alves C.J., Nobre V.M.T., Athayde A.C.R., Gomes A.A. \& Lima E.F. 2003. Doenças dos ruminantes e eqüinos no semi-árido da Paraíba. Semi-árido em Foco 1(1):58-60.

Riet-Correa F., Medeiros R.M.T. \& Dantas A.F.M. 2006. Plantas Tóxicas da Paraíba. UFCG/CSTR/HV, Sebrae/PB, Patos. 58p.

Tokarnia C.H., Döbereiner J., Peixoto P.V., Barbosa J.D., Brito M.F. \& Silva M.F. 2007. Plantas Tóxicas da Amazônia. 2ª ed. INPA, Manaus. 96p.

Tokarnia C.H., Brito M.F., Barbosa J.D., Peixoto P.V. \& Döbereiner J. 2012. Plantas Tóxicas do Brasil para Animais de Produção. 2a ed. Editora Helianthus, Rio de Janeiro.

Tortelli P.F., Barbosa J.D., Oliveira C.M.C., Dutra M.D., Cerqueira V.D., Oliveira C.A., Riet-Correa F. \& Riet-Correa G. 2008. Intoxicação por Ipomoea asarifolia em bovinos e ovinos na Ilha de Marajó. Pesq. Vet. Bras. 28(12):622-626. 